\title{
Emerging Therapeutic Approaches to Hepatocellular Carcinoma
}

\author{
Timothy M. Pawlik, MD, MPH \\ Department of Surgery, The Johns Hopkins University School of Medicine, Baltimore, MD
}

Hepatocellular carcinoma (HCC) is one of the most common solid malignancies worldwide, and its incidence is dramatically increasing in the United States. Unlike many other malignancies, treatment of HCC often involves management of two disease processes: liver cancer and cirrhosis. In addition to tumor-specific factors such as tumor size, number, and location, the surgeon also has to consider liver specific factors such as degree of fibrosis/ cirrhosis, extent of portal hypertension, and the size of the future liver remnant. As such, therapy for HCC is complex and can involve resection, transplantation, interventional radiology-based liver-directed therapies, and emerging systemic chemotherapeutic and targeted agents. To successfully treat this group of patients, surgical oncologists should be aware of the many and varied approaches to treating patients with HCC. Specifically, surgical oncologists need to know not only how to select patients and perform hepatic resection in appropriate patients with $\mathrm{HCC}$, but also be familiar with the controversy surrounding

Acknowledgment: This educational review series, "Emerging Therapeutic Approaches to Hepatocellular Carcinoma," has been supported by an educational grant from Onyx Pharmaceuticals/Bayer Healthcare Pharmaceuticals.

Annals of Surgical Oncology educational reviews represent the journal's commitment to the peer review and publication of highquality research necessary to define the safety, toxicity, or effectiveness of potential therapeutic agents compared with conventional alternatives.

This Educational Review Series may include information regarding the use of medications that may be outside the approved labeling for these products. Physicians should consult the current prescribing information for these products. Authors of Annals of Surgical Oncology educational reviews are provided at the time of article solicitation with this statement regarding off-label pharmaceutical information and research.

(C) Society of Surgical Oncology 2010

T. M. Pawlik, MD, MPH

e-mail: tpawlik1@jhmi.edu management of early HCC and identify those factors that may make one therapeutic modality preferable compared with others. In addition, surgical oncologists need to be familiar with the role of intra-arterial regional therapy as "destination" therapy for unresectable HCC, as well as bridge therapy before transplantation/resection. Finally, surgical oncologists need to be aware of the emerging role of systemic chemotherapy for stage IV HCC, as well as its potential future role as adjuvant therapy. In this issue of Annals of Surgical Oncology, a series of review articles have been solicited to provide evidence-based data to equip the practicing surgical oncologist with the knowledge to understand these complex treatment issues. ${ }^{1-4}$

In the first article, surgical resection of HCC is addressed with a particular focus on patient selection, preoperative considerations, and perioperative management of patients. ${ }^{1}$ The selection of HCC patients for surgical resection continues to evolve and expand with advances in technology and improved perioperative care of the patient with cirrhosis. Specifically, preoperative portal vein embolization has made resection possible for a subset of patients who otherwise might not have been considered surgical candidates as a result of an anticipated small liver remnant. However, resection of large or multifocal HCC, as well as resection in those patients with portal hypertension, remains controversial. In the second article, another controversy in the management of HCC is addressed: therapy for early-stage $\mathrm{HCC}$ in patients with well-compensated cirrhosis. ${ }^{2}$ Among patients with a solitary tumor $\leq 5 \mathrm{~cm}$ in size or three or fewer tumors each $\leq 3 \mathrm{~cm}$ in size and no evidence of gross vascular invasion, the role of resection versus transplantation versus ablation is examined. Although the ideal treatment strategy for this subset of patients remains controversial, data on the relative indications and merits of each therapeutic approach are presented.

In the third article, issues related to intra-arterial therapies for inoperable HCC are discussed. ${ }^{3}$ Unfortunately, many patients with $\mathrm{HCC}$ will present with advanced disease and will not be candidates for either surgical resection 
or transplantation. Catheter-based locoregional administration of intra-arterial therapeutic and/or embolic agents, therefore, often represents the main therapeutic option. The field of catheter-based therapy continues to advance at a rapid pace with emerging new agents that can be delivered intra-arterially, including drug-eluding beads, as well as yttrium-90 and iodine-131-labeled lipiodol.

The fourth article addresses the emerging role of systemic therapy for HCC. ${ }^{4}$ For decades, systemic and hormone therapies for $\mathrm{HCC}$ have failed to demonstrate improved survival for patients with HCC. However, with the advent of the Sorafenib HCC Assessment Randomized Protocol (SHARP) trial, a new era of molecular-targeted therapy has begun. The final review focuses on the emerging role of chemotherapy with a particular emphasis on molecular-targeted approaches.

It is our hope that the series of articles published in this issue of Annals of Surgical Oncology will serve as an educational resource and a worthwhile synopsis of the current state-of-the-art therapeutic approaches to HCC. In turn, these articles can, we hope, frame the important issues, highlight the need for a multidisciplinary approach, and inform the surgical oncologist as we treat this complex group of patients.

Author disclosure: Dr. Pawlik has provided consultant services to Onyx Pharmaceuticals/Bayer Healthcare Pharmaceuticals.

\section{REFERENCES}

1. Truty MJ, Vauthey JN. Surgical resection of high-risk hepatocellular carcinoma: patient selection, preoperative considerations, and operative technique. Ann Surg Oncol. doi:10.1245/s10434-0100976-5.

2. Jarnagin WR. Management of small hepatocellular carcinoma: a review of transplantation, resection, and ablation. Ann Surg Oncol. doi:10.1245/s10434-010-0978-3.

3. Liapi E, Geschwind JF. Intra-arterial therapies for hepatocellular carcinoma: where do we stand? Ann Surg Oncol. doi:10.1245/ s10434-010-0977-4.

4. Zhu AX. Systemic treatment of hepatocellular carcinoma: dawn of a new era? Ann Surg Oncol. doi:10.1245/s10434-010-0975-6. 